\title{
8
}
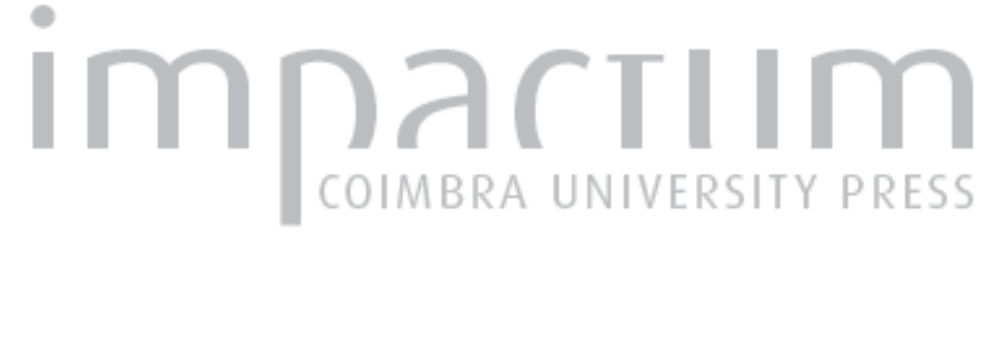

[Recensão a] M. a A. Duran López \& R. Caballero Sánchez, Plutarco. Obras morales y de costumbres (Moralia) XL Tratados platónicos.

\section{Tratados antiestoicos}

Autor(es): Macías, Cristóbal

Publicado por: International Plutarch Society

URL

persistente:

URI:http://hdl.handle.net/10316.2/37632

DOI:

DOI:http://dx.doi.org/10.14195/0258-655X_4_8

Accessed : $\quad$ 26-Apr-2023 15:13:17

A navegação consulta e descarregamento dos títulos inseridos nas Bibliotecas Digitais UC Digitalis, UC Pombalina e UC Impactum, pressupõem a aceitação plena e sem reservas dos Termos e Condições de Uso destas Bibliotecas Digitais, disponíveis em https://digitalis.uc.pt/pt-pt/termos.

Conforme exposto nos referidos Termos e Condições de Uso, o descarregamento de títulos de acesso restrito requer uma licença válida de autorização devendo o utilizador aceder ao(s) documento(s) a partir de um endereço de IP da instituição detentora da supramencionada licença.

Ao utilizador é apenas permitido o descarregamento para uso pessoal, pelo que o emprego do(s) título(s) descarregado(s) para outro fim, designadamente comercial, carece de autorização do respetivo autor ou editor da obra.

Na medida em que todas as obras da UC Digitalis se encontram protegidas pelo Código do Direito de Autor e Direitos Conexos e demais legislação aplicável, toda a cópia, parcial ou total, deste documento, nos casos em que é legalmente admitida, deverá conter ou fazer-se acompanhar por este aviso. 
PLOUTARCHOS, n.S. Scholarly Journal of 18

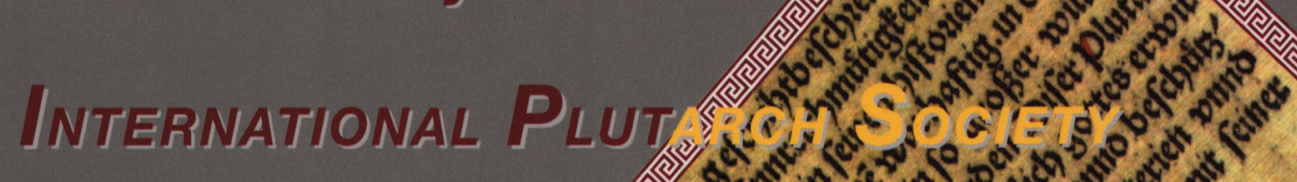

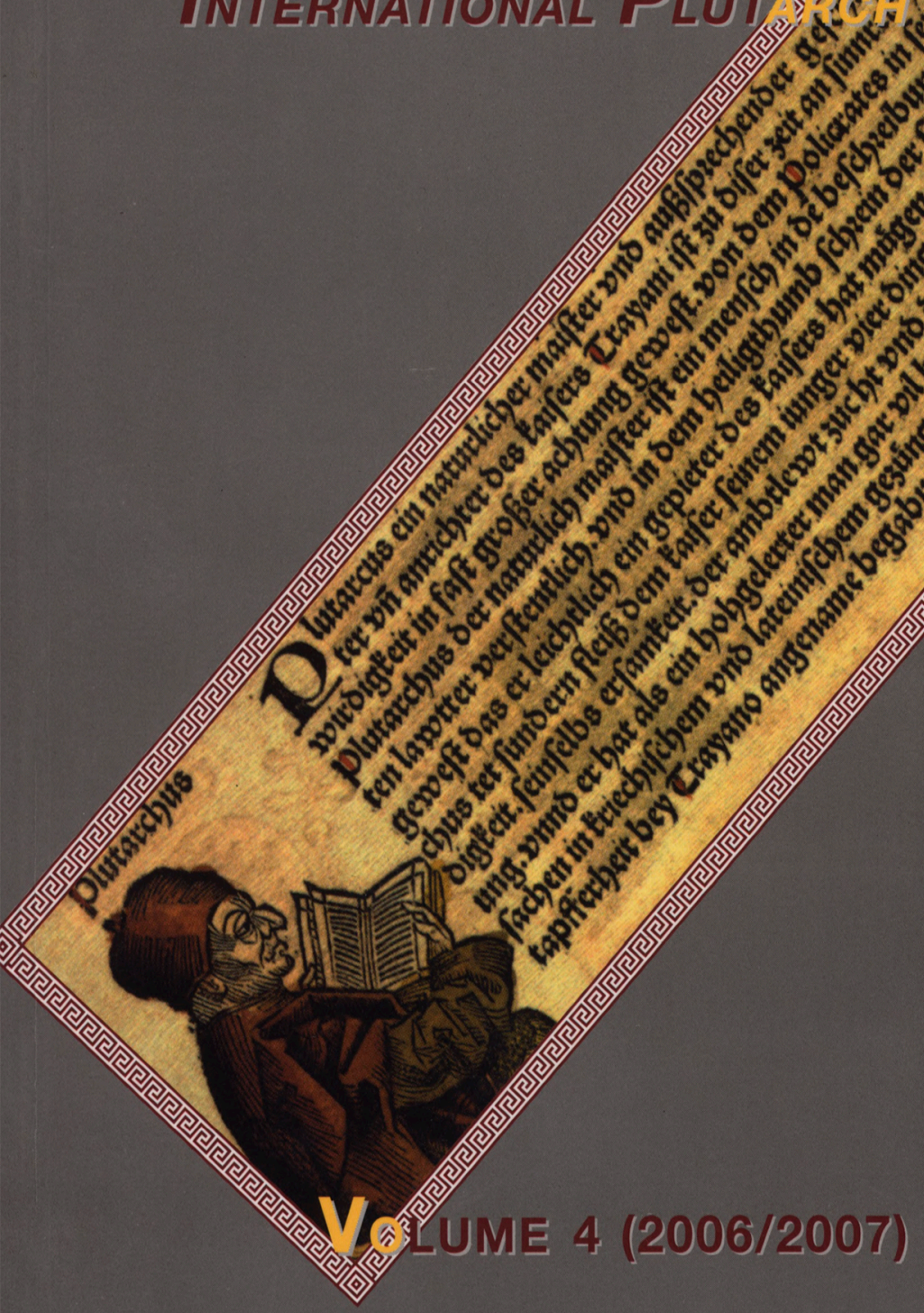

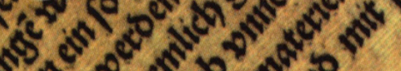

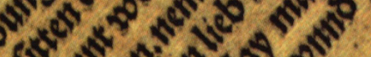

$120^{2} 0^{2} x^{2} x^{2}$

$4 \sin ^{\circ} \cos ^{\circ}$

singes

$0^{2}+0^{2} \mathrm{~s}^{\circ}$

$a^{5} x^{2}$

$s^{5}+\omega^{\circ}$

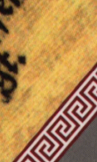

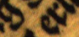

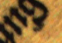


radas, notablemente moralizante y moralizado (al modo de Menandro), en tanto que deplora la procacidad verbal del humor burlón, escarnecedor, que representa Aristófanes.

Por su parte, Helena Rodríguez facilita las traducciones de tratados políticos que, en el inventario plutarqueo, observan una intención eminentemente pedagógica y práctica. He aquí opúsculos vertidos por primera vez a nuestra lengua; y si la temática de los mismos es parcialmente convergente, no puede indicarse lo mismo de su transmisión, estilo y notoriedad literaria. El primero de ellos \{Sobre la necesidad de que el filósofo converse con los gobernantes) presenta un texto sensiblemente deturpado en la tradición manuscrita; el segundo (A un gobernante falto de instrucción) da la impresión de no hallarse acabado; y el tercero \{Sobre si el anciano debe intervenir en política) pertenece a un período avanzado de la vida de Plutarco: precisamente aquí nuestro polígrafo despliega la versatilidad de sus recursos literarios, sin parquedad de anécdotas, citas, comparaciones de toda laya. Debe indicarse que la autora, tanto en la versión anotada como en la introducción que las precede, acierta a valorar con elegancia la singularidad de cada tratado mediante claros prólogos de presentación y traducciones notables.

Finalmente, Carlos Alcalde, quien ya cuenta con experiencia de traducción en las Vidas de Plutarco, se ajusta aquí a ensayos de naturaleza asimismo política; y priman en estos escritos las reflexiones de condición teorico-práctica. Ahí está, por ejemplo, el conocido tratado Consejos políticos, una de las más brillantes aportaciones del queronense al tema que, entre nosotros, ya estudió y tradujo F. Gaseó: aquí Plutarco refiere personalmente admoniciones de importancia a Menémaco de Sardes, un joven en quien se ha despertado la vocación política. La traducción de Alcalde es fluida y bien anotada. Tras el brevísimo --y a menudo juzgado espurio- Sobre la monarquía, la democracia y la oligarquía, constan ciertas reflexiones de cariz socioeconómico que Plutarco articula en una sinopsis de índole manifiestamente propedéutica y moral $\{$ La inconveniencia de contraer deudas): y es que Plutarco insta a que las clases acomodadas sean parsimoniosas y renuncien a la petición desmesurada de préstamos. Todo ello queda conformado, en la version de Alcalde, mediante una prosa ágil y cómodamente legible.

VICENTE RAMON \& ANA VICENTE Universidad de Zaragoza

JYT A. DuRan LÓPEZ \& R. CABAllero SÁNCHEZ, Plutarco. Obras morales $y$ de costumbres (Moralia) XL Tratados platónicos. Tratados antiestoicos, Madrid, G redos, 2004, 522 pp. ISBN 84-249-2715-X.

La "Biblioteca Clásica Credos" publicó en 2004, como número 322 de esta prestigiosa colección, el volumen XI de los Moralia de Plutarco, constituido por sus Tratados platónicos y los Tratados antiestoi$c o s$, a cargo de los profesores de la Universidad de Málaga Ma. A. Durán y R. Caballero, respectivamente.

Bajo el título de Tratados platónicos, la tradición nos ha legado dos escritos de Plutarco sobre filosofía platónica, las Cuestiones platónicas, muy breve, y el tratado Sobre la generación del alma en el "Timeo", que está seguido por el Epitome del tratado "Sobre la generación del alma en el "Timeo "”, que no es obra del autor griego, por lo que no aparece en el Catálogo de Lamprias, aunque sí en el de Máximo Planudes. Entre los tratados platónicos que menciona el primero de los catálogos figuran otros no conservados, a saber, los núms. 66, $67,68,70$ y 221 , que trataban respectivamente de la generación del Universo según Platón, de la ubicación de las Ideas, de cómo 
participa la materia de las ideas, del Teages y de la teleología platónica.

Por su parte, como Tratados antiestoicos nos han llegado tres opúsculos polémicos, Las contradicciones de los estoicos, Los estoicos dicen más disparates que los poetas y Sobre las nociones comunes, contra los estoicos (núms. 76, 79 y 77 del Catálogo de Lamprias), dirigidos casi exclusivamente contra la doctrina estoica tal como aparece en Crisipo, uno de los representantes más cualificados del estoicismo antiguo. Además de estos tres pequeños tratados, sabemos que Plutarco escribió otros tratados antiestoicos que, por desgracia, no conservamos: Sobre lo que depende de nosotros, contra los estoicos (núm. 154); Sobre la justicia, contra Crisipo (núm. 59); Sobre la experiencia común, contra los estoicos (núm. 78); Causas de las oscilaciones (doctrinales) de los estoicos (núm. 149); Sobre el primer consecuente, contra Crisipo (núm. 152).

Respecto a los tratados de índole platónica, es sabido que, aunque Plutarco militó en las filas del platonismo, su actividad se ejerció más junto a la Academia que en las coordenadas de ésta. Esto significa, por un lado, que en la obra del de Queronea lo platónico no se reduce a los tratados mencionados, sino que el maestro griego ocupa una posición de relieve también en otras obras que no están dedicadas a él, como los tratados isis y Osiris, Sobre el demon de Sócrates o Sobre la $E$ de Delfos, sin olvidar las numerosas citas y alusiones presentes en toda la obra plutarquea; por otro, su militancia platónica no le hace olvidar los numerosos desacuerdos que mantiene con los platónicos de su tiempo, con los que no quiere entrar en polémicas, pero que cuando lo hace, sobre todo en el tratado sobre la generación del alma, no duda en rechazar con contundencia las incongruencias que éstos atribuyen a Platón. Asimismo, por los tratados platónicos conservados queda clara la importancia que para el Queronense tiene el Timeo, hecho debido, al menos en parte, al interés de su época por los aspectos escatológicos, cosmológicos y metafísicos - no olvidemos que durante los primeros siglos de nuestra era, entre otras cosas, se va consolidando el sincretismo religioso de los dioses importados de Oriente y Egipto con los dioses griegos-y al deseo de desentrañar las dificultades inherentes a este diálogo, lo cual sitúa a Plutarco en la larga tradición de exégetas y comentaristas del Timeo que arranca con Aristóteles y Crantor y llega hasta finales de la Antigüedad con Calcidio y Proclo.

Entre los Tratados platónicos, el de Cuestiones platónicas (pp. 32-85) reúne diez breves comentarios a puntos concretos del pensamiento platónico, que pueden centrarse en un único pasaje o bien pueden enlazar varios, intentando explicar unos con la ayuda de los otros. En ellos no se discuten puntos fundamentales de la doctrina del maestro, sino "curiosidades" marginales que Platón no consideró oportuno desarrollar in extenso.

El carácter puntual de las Cuestiones las acerca a otros escritos plutarqueos como las Charlas de sobremesa y los diferentes Aitíai. Frente a los escritos de carácter simposíaco, las Cuestiones carecen de introducción y de conclusión, y tampoco sabemos por qué criterio se seleccionó un tema y no otro. Lo único que tienen en común es que se trata de cuestiones tratadas o aludidas en alguno de los diálogos de Platón, aunque es perceptible la importancia que se concede a las cuestiones relacionadas con el Timeo.

Formalmente, las Cuestiones se definen como zetémata, término técnico usado en principio en el marco de la explicación de los poemas homéricos, para aludir a la exposición de una dificultad en una palabra o verso, seguida de una propuesta de solución. En el caso del texto plutarqueo, el tema que se va a tratar se plantea en forma de pregun- 
ta (o cadena de preguntas), formulada de modo impersonal: "Pues bien, ¿con qué objetivo dividió el conjunto en segmentos desiguales y cuál de los segmentos es mayor, el inteligible o el perceptible?" (Cuestión III, p. 42); a ésta le siguen dos o más respuestas. A pesar de ello, hay cuestiones que quedan sin respuesta, como la I o la VI.

Respecto al contenido, las Cuestiones se pueden agrupar entre las que intentan zanjar la polémica sobre la interpretación de algún punto de la doctrina platónica — polémicas suscitadas a veces por problemas de crítica textual, como la Cuestión III y en parte también en la VIII; o las que tratan de explicar alguna metáfora o expresión - como en la II, "¿Por qué será que al dios supremo lo llamó padre y creador de todas las cosas?".

En fin, la traducción de las Cuestiones como la del tratado Sobre la generación del alma sigue la edición de C. Hubert-H. Drexler.

Por su parte, el tratado Sobre la generación del alma en el "Timeo" (pp. 108-174), un híbrido entre carta familiar y lo que hoy llamaríamos artículo científico, se compuso como respuesta a una solicitud de los hijos de Plutarco para que resumiera la doctrina platónica del alma, cuestión a la que hasta ese momento no había dedicado un tratado sistemático. El hecho de responder a una petición de sus hijos apoya la tesis de que se trata de una obra de madurez.

Como deja entrever el título, Plutarco no aborda todas las cuestiones que suscitaba el Timeo. Su tema principal es el del alma del Mundo, al que añadirá una exposición sobre los números. En fin, hay puntos que no trata, pero sobre los cuales su postura queda clara, como el carácter mítico o no del Timeo. A este respecto, Plutarco rechaza cualquier clase de interpretación alegórica y, en concreto, que la generación del alma y del cuerpo del Mundo sea un mero recurso expositivo.

En cuanto a su estructura, establecida en sus líneas generales por Hershbell, tras el preámbulo, comprende dos partes perfectamente diferenciadas, precedida cada una por la cita literal del pasaje del diálogo que Plutarco pretende explicar. La primera, que abarca desde 1012B, con la cita de Timeo $35 \mathrm{a}$, hasta aproximadamente $1027 \mathrm{~A}$, está dedicada al alma según Platón y es la que responde directamente a la petición de sus hijos; la segunda, que comienza en 1027B con la cita de Timeo 35b-36b y se extiende hasta el final del opúsculo en 1030C, está dedicada a los números, y en ella se da cumplida información sobre las especulaciones aritméticas, musicológicas y astronómicas desarrolladas por Platón en Timeo 35b-36c.

En fin, el mal llamado Epitome (pp. 177183) es una copia de dos capítulos del tratado Sobre la generación del alma, precedidos de una introducción. El epítome, anónimo, no es, como ya se ha dicho, de Plutarco, al cual curiosamente se alude siempre mediante el anafórico o con verbos en tercera persona. Además, el texto presenta un buen número de errores o deficiencias, ya señaladas por la crítica, que parecen demostrar que su anónimo autor no entendió el texto que tenía delante. Entre sus aspectos positivos, en algunos casos conserva palabras omitidas en todos los manuscritos o bien nos permite restituir la forma correcta de textos que en todos los manuscritos aparecían con faltas, de lo cual se concluye que el autor del epítome empleó un ejemplar diferente a los que se utilizaron para copiar el texto del tratado.

En cuanto a los Tratados antiestoicos, Plutarco los escribió como un modo de marcar distancias con la filosofía dominante en su época, el estoicismo, para, de ese modo, afirmar sus propias convicciones platónicas. Además, igual que el platonismo, directa o indirectamente, llena toda su obra, la polémica antiestoica también está presente de un modo o de otro tanto en sus disertaciones y 
diálogos, como en sus tratados ético-didácticos y en sus obras técnicas de filosofía. Pero quizás lo que más sorprenda al lector moderno no especialista es que Plutarco decida dirigir toda su artillería dialéctica contra las doctrinas del Pórtico más antiguo, el de Crisipo, en vez de contra los representantes contemporáneos de la escuela, como Epicteto. No obstante, a modo de justificación, hay que advertir que el núcleo fundamental del estoicismo de época romana había sido ya fijado por el estoicismo antiguo, en particular por Crisipo, lo cual permite entender la postura del de Queronea.

Asimismo, los opúsculos que componen los Tratados antiestoicos son tratados de polémica filosófica especializados, por lo que parecen dirigidos a un público minoritario, buen conocedor de las doctrinas estoicas y familiarizado con el rico debate suscitado entre las diversas escuelas en esta época. Esto, sin duda, es lo que justifica el sesgo técnico que a veces alcanza la exposición, que a un lector moderno se le puede antojar prolijo y complejo.

En cuanto al primero de los tratados, Las contradicciones de los estoicos (pp. 212339), el segundo más extenso de los tres textos de polémica antiestoica conservados, en él Plutarco pretendía poner de relieve las contradicciones internas de la doctrina de Crisipo, desmintiendo de esta forma la supuesta coherencia y sistematicidad de la filosofía de los estoicos, de la que tan orgullosos se mostraban éstos. Es decir, estamos ante un esfuerzo polemista de alcance muy limitado, algo que el propio Plutarco se encarga de advertir en ocasiones al lector.
Respecto a la cronología absoluta ${ }^{1}$ de composición del tratado, Babut ha sugerido situarlo en el periodo de madurez de la vida de Plutarco, estableciendo como terminus ante quem la composición de los tratados antiepicúreos, de forma que Las contradicciones de los estoicos pertenecerían a la década de los 80 o 90 del primer siglo de nuestra era.

En cuanto a su estructura, como ha demostrado Cherniss, no es cierto que ésta se base en la mera yuxtaposición o acumulación de capítulos sin aparente conexión entre sí , sino que se han puesto enjuego otros criterios estructurales como la asociación de ideas o la inclusión de digresiones, que no son ajenos a los tratados filosóficos desde los tiempos más remotos.

De hecho, como puede deducirse del detallado esquema que el profesor Caballero desarrolla en las pp. 199 a 204, la obra presenta tres partes perfectamente diferenciadas: A. I-III. Introducción (teoría y práctica de la actividad filosófica): B. IV-VII. Ética de Crisipo; C. V1II-XII. Física de Crisipo (donde entraría su teodicea, la teología y sus doctrinas sobre el Destino). Esto significa que, aunque no se ha dedicado una sección autónoma a la Lógica, el orden de los temas se ajusta bastante bien a la división estoica de las partes de la filosofía.

Sobre las posibles fuentes, una vez descartado que nos encontremos ante una mera acumulación de materiales inconexos y superadas las reticencias que había sobre la fiabilidad de Plutarco como doxógrafo de Crisipo, hoy día se considera que Plutarco

Carecemos de argumentos definitivos para establecer una cronología relativa entre los distintos opúsculos de la polémica antiestoica.

2

De hecho, como apunta el profesor Caballero (p. 199), el hecho de carecer de un epílogo como coronación de la obra ha podido contribuir a reforzar esta imagen de descuido o negligencia estructural. 
manejó directamente la obra de éste, la cual demuestra conocer bien, como confirma la abundancia de citas literales y paráfrasis sémi literales repartidas por su obra, con indicación expresa del libro consultado en cada caso. Asimismo, la agresividad que a menudo muestra en su polémica y el tipo de argumentos que esgrime se explican porque, primero, la confrontación dialéctica entre escuelas ya preveía esa agresividad que a menudo sorprende en Plutarco; en segundo lugar, se podrían explicar como exigencias del género las aparentes distorsiones de las doctrinas estoicas presentes en sus panfletos. Otra cosa bien distinta son los errores imputables al autor, que también los hay.

En fin, la traducción de Las contradicciones de los estoicos, al igual que las de los otros dos tratados antiestoicos, sigue en lo esencial la edición de Cherniss de los Moralia, XIII, 2, Londres-Cambridge (Massachusetts, 1976).

El segundo opúsculo de la polémica antiestoica lleva el expresivo título de Los estoicos dicen más disparates que los poetas y es el más breve de los tres (pp. 345-350). En él Plutarco, en son de burla, compara las más célebres doctrinas de los estoicos con las más disparatadas creaciones de la imaginación poética y mítica. $\mathrm{Y}$ aunque en los códices el título viene precedido por la fórmula "Resumen de" — con lo que estaríamos ante un compendio de una obra más extensa perdida-, la lectura del texto parece dejar bien claro que no estamos ante ningún resumen, sino más bien ante el extracto literal de una parte del texto original.

El tema que vertebra las mordaces observaciones de Plutarco es el de la imagen tópica del sabio estoico: el hombre impasible ante las desgracias, capaz de pasar del vicio a la virtud y que en medio de la miseria se reivindica como el más bello, rico y próspero.

En fin, nada seguro podemos decir sobre la cronología de esta obrita, salvo que, quizás, es anterior a Sobre las nociones comunescontra los estoicos.

El tercero de los tratados antiestoicos es, precisamente, el de las "nociones comunes", que acabamos de mencionar, que además es el más extenso de los tres (pp. 368-506). Presenta la forma de un diálogo, más aparente que reaf, entre un supuesto maestro, Diadúmeno, trasunto literario del propio autor, con un discípulo, cuyo nombre no conocemos, que aparece angustiado por los ataques que los estoicos dirigían contra los académicos, antiguos y coetáneos, a los que acusaban de intentar minar la filosofía de Crisipo acabando con las nociones comunes, aquellas nociones universales que, ancladas en los sentidos, constituyen la base más sólida del conocimiento y la ciencia.

El modo de neutralizar tales ataques es la fírme convicción que presenta el maestro, como buen académico, de que ningún sistema doctrinal sólido puede levantarse sobre el mundo fenoménico; es decir, que las nociones comunes, basadas en los sentidos, no pueden por sí mismas ser garantía de ningún conocimiento seguro. Negada así la

Es posible que conociera a Crisipo durante sus años de formación en Atenas o, como sugiere el profesor Caballero, que lo consultara en su propia biblioteca particular o en la de la Academia de Queronea.

4

Como el hecho de silenciar parte de la doctrina criticada o el no contextualizar adecuadamente los pensamientos de Crisipo.

5

Pues a partir del cap. $4^{\circ}$ la intervención de Diadúmeno se convierte en un monólogo continuo, pocas veces interrumpido por su interlocutor para pedirle aclaraciones terminológicas o conceptuales o que se detenga en un argumento o que pase a otro. 
validez de tales nociones, el propio ataque de los estoicos contra los académicos en este punto queda desactivado.

Respecto a la cronología del diálogo, valga lo dicho para el caso de Las contradicciones de los estoicos.

En cuanto a su estructura (pp. 354-359), el diálogo se compone de una introducción (I), de dos secciones casi idénticas dedicadas respectivamente a la ética (II-VI) y a la física (VIII-XII) de los estoicos, con una sección Vil que sirve de tránsito entre ambas. De este modo, su organización interna es muy similar al tratado de Las contradicciones, incluyendo el hecho de que ninguno de los dos trata el tema de la Lógica. La diferencia más significativa radica en que en el tratado de las nociones comunes lo que se dice de la Teodicea se incluye en la parte dedicada a la ética.

En fin, se ha discutido la finalidad última de este diálogo, apuntándose en este sentido el hecho de que quizás se escribió para poner de relieve las paradojas de los estoicos (como el tratado de Cicerón), es decir, aquellos puntos de su doctrina que atentaban contra el sentido común. Sin embargo, si partimos de la base de que para los estoicos las "nociones comunes" no siempre coinciden con las opiniones admitidas comúnmente por la humanidad - sino que, al contrario, éstas eran consideradas por ellos como meros prejuicios y errores, fruto de una educación perversa y de un sistema de valores que prima, por ejemplo, la riqueza y la fama sobre bienes verdaderos, como la virtud del mendigo- y que esto lo sabe Plutarco, el fin último del tratado sería denunciar las contradicciones que se perciben entre las principales nociones comunes de los estoicos y las doctrinas éticas y físicas que de ellas se desprenden.

De otro lado, en el trabajo de los profesores Durán y Caballero, en el que hay que valorar en primer lugar el extraordinario y encomi ab le esfuerzo que han hecho por acercar a los lectores españoles el texto plutarqueo de estos opúsculos, respetando escrupulosamente el original y tratando de buscar siempre el término español que mejor recoja la palabra o expresión griega correspondiente (además del hecho de que Las contradicciones de los estoicos y Sobre las nociones comunes es la primera vez que se vierten a nuestra lengua), no podemos dejar de mencionar la importancia que en este tipo de obras tienen para un lector moderno las notas al pie, sin olvidar las introducciones generales y las particulares que preceden a la traducción de cada opúsculo. Así lo ponen de relieve ambos traductores: la profesora Durán (pp. 19-20), enumerando la tipología de las notas aclaratorias que se ha visto obligada a incluir para acercar a un lector no necesariamente iniciado cuestiones de índole filosófica o científica, entre ellas, las que sirven para identificar los pasajes de los diálogos platónicos citados por Plutarco o las obras de los demás filósofos aludidos, las que recogen referencias a otros pasajes del corpus platónico relativos a la misma cuestión, asimismo las referencias a otros pasajes del cuerpo plutarqueo que pueden ayudar a comprender mejor el pasaje en cuestión y las notas procedentes de la inmensa bibliografía generada por el pensamiento y la obra de Platón y Plutarco - de la cual, no obstante, sólo se menciona la que realmente se ha manejado-; por su parte, el profesor Caballero, advirtiendo (p. 190) que ha tenido que añadir numerosas y extensas notas a la traducción, para restituir al lector moderno todo aquello que Plutarco hurtaba o dejaba implícito a sus lectores, en concreto, el contexto filosófico original de las doctrinas estoicas atacadas, con idea de que el lector pueda formarse su propio juicio.

CRISTÓBAL MACÍAS Universidad de Málaga 